\title{
Barriers to Follow-Up Colonoscopy After Positive FIT or Multitarget Stool DNA Testing
}

\author{
Gregory S. Cooper, MD, Ashley Grimes, MS, James Werner, PhD, Shufen Cao, PhD, \\ Pingfu Fu, PhD, and Kurt C. Stange, $M D, P h D$
}

Background: Fecal immunochemical testing (FIT) and multi-target stool DNA testing (mt-sDNA) are recommended colorectal cancer screening options but require follow-up with colonoscopy to determine the source of a positive result. We performed a retrospective analysis in an academic health system to determine adherence to colonoscopy in these patients.

Methods: We identified all patients aged 40 years and older with at least 1 primary care visit who had a positive FIT or mt-sDNA between January 2016 and June 2018. We identified receipt of colonoscopy within 6 months of the positive test and reviewed medical records to determine reasons for lack of colonoscopy.

Results: We identified 308 eligible patients with positive FIT and 323 with positive mt-sDNA. Some patients with positive FIT (46.7\%) and patients with positive mt-sDNA (71.5\%) underwent colonoscopy within 6 months, and time to colonoscopy was also shorter with mt-sDNA (hazard ratio, 1.83; 95\% CI, 1.48-2.25). These differences remained in a multivariable model adjusting for patient characteristics. Among patients without colonoscopy after positive FIT, 1 or more system, provider, and patient-related barriers were identified in 32.1\%, 57.6\%, and 36.3\%, respectively. Among patients without colonoscopy after positive mt-sDNA, corresponding frequencies were $30.4 \%, 43.5 \%$, and $57.6 \%$, respectively.

Conclusions: Follow-up colonoscopy was higher for mt-sDNA than FIT, which could be due in part to preselection by clinicians and/or patients. Among patients who did not follow-up, provider and system factors were as frequently encountered as patient factors. These findings reinforce the need for multi-level interventions to improve follow-up. (J Am Board Fam Med 2021;34:61-69.)

Keywords: Cancer Screening, Colonoscopy, Colorectal Cancer, Mass Screening, Occult Blood

\section{Introduction}

Colorectal cancer (CRC) is the second most fatal cancer in the United States ${ }^{1}$ but the majority of cancers are preventable through the use of screening beginning at age 45 or 50 years in the average-risk population. ${ }^{2-4}$ Most guidelines recommend a choice of screening options in addition to colonoscopy,

This article was externally peer reviewed.

Submitted 6 July 2020; revised 11 August 2020; accepted 12 August 2020.

From the Division of Gastroenterology, University Hospitals Cleveland Medical Center, Cleveland OH (GSC, AG); Center for Community Health Integration and Department of Family Medicine, Case Western Reserve University, Cleveland $\mathrm{OH}$ (JW, KCS); Department of Population and Quantitative Health, Case Western Reserve University, Cleveland OH (SC, $\mathrm{PF}$; and the Case Comprehensive Cancer Center, Cleveland $\mathrm{OH}$ (GSC, JW, PF, KCS).

Funding: Supported by a pilot grant from the Case Comprehensive Cancer Center (P30 CA43703).

Conflict of interest: None.

Corresponding author: Gregory S. Cooper, MD, Division of Gastroenterology, University Hospitals Cleveland Medical including less invasive screening methods that are fecal based. ${ }^{2-4}$ The 2 most commonly used stool based methods are the fecal immunochemical test (FIT), which detects globin from human hemoglobin; and a multitarget stool DNA panel (mt-sDNA), which includes 2 aberrant DNA methylation markers, K-Ras oncogene as well as FIT (Cologuard, Exact Sciences, Inc., Madison, WI) ${ }^{5}$ (Table 1). Although providing noninvasive screening options such as FIT and mt-sDNA are likely to improve overall adherence to CRC screening, these tests are only effective if patients adhere to follow-up colonoscopy if the tests are positive. The US Multisociety Task Force on Colorectal Cancer has set a benchmark of $80 \%$ adherence with colonoscopy for positive tests. ${ }^{6}$

Center, 11100 Euclid Avenue, Wearn 244, Cleveland, OH 44106-5066 (E-mail: gregory.cooper@uhhospitals.org). 
Table 1. Characteristics of FIT and mt-sDNA Tests

\begin{tabular}{|c|c|c|}
\hline Characteristic & FIT & Mt-sDNA \\
\hline Measures stool hemoglobin & Yes & Yes \\
\hline Measures genomic markers & No & Yes \\
\hline Approved as screening test & Yes & Yes \\
\hline Labeling restricted to average risk patients & No & Yes \\
\hline Recommended interval & 1 year & 3 years \\
\hline Kit distribution & In-person or mail from local lab & Mail from external site \\
\hline Kit return & In person or mail to local lab & Mail to external site \\
\hline Reminder calls to return & No & Yes \\
\hline Medicare out-of-pocket cost & $\$ 0$ & $\$ 0$ \\
\hline Medicare reimbursement & $\$ 22$ & $\$ 483$ \\
\hline
\end{tabular}

FIT, fecal immunochemical testing.

Compliance with follow-up colonoscopy after a positive FIT is as high as $90 \%$ in certain groups of privately insured patients, but a multicenter consortium ${ }^{7}$ found follow-up rates of as low as $40 \%$ to $50 \%$. Other studies have found follow-up to be $40 \%$ to $60 \%$ in Veterans Affairs (VA) centers ${ }^{8-10}$ and $69 \%$ in a large health maintenance organization. ${ }^{11}$ With the exception of one study of approximately 50 patients in a multispecialty group practice, ${ }^{12}$ similar data on frequency of follow-up colonoscopy after a positive mt-sDNA tests are not available. Furthermore, delays in time to colonoscopy after positive FIT testing are associated with risk of CRC and advanced stage disease. ${ }^{13-14}$ In fact, each month delay that occurs between a positive FIT and a follow-up colonoscopy is associated with a $0.1 / 1000$ person increase in cancer incidence. $^{15}$

There are at least 3 potential levels for lack of follow-up for a positive stool test: patient, provider, and system, and all 3 have been implicated in previous studies. ${ }^{9,10,16-18}$ Moreover, these 3 levels are intimately connected as patient may fail to attend their colonoscopy appointment if the provider does not discuss the patient's screening results and the reasons why colonoscopy is necessary. The goal of our study was to use electronic medical record data from a large vertically integrated health care system (ie, hospital acquired physician practices) to evaluate the frequency of follow-up colonoscopy after a positive FIT or mtsDNA test and to determine underlying reasons for lack of follow-up. Our study is one of the few that were performed in an academic health care system and included more than one noninvasive screening test.

\section{Methods}

\section{Study Design and Population}

We performed a retrospective cohort study among patients in a large, vertically integrated health care system in the Midwest. Approval for the study was obtained from the Institutional Review Board. Using the ambulatory electronic medical record (AEMR), we identified men and women aged 40 years and older with either a positive FIT or mtsDNA between January 2016 and June 2018. Both tests required a provider order to be distributed. All FITs were distributed to patients who then returned the kit to the hospital laboratory where they were run centrally using a cutoff of $50 \mu \mathrm{g} / \mathrm{gram}$ stool as a positive test. The FIT (OC-Auto Sensor Diana, Polymedco, Cortlandt, NY) test results were sent directly to the electronic task lists of the ordering provider where they were verified. The mtsDNA kits (Cologuard, Exact Sciences, Inc.) were mailed to the patients' home. Stool samples were then mailed directly to Exact Sciences where they were reported as a single positive or negative result. Providers were sent the results, which were then scanned into the AEMR and for all positive mtsDNA results, providers were also telephoned to reinforce the positive tests. All verification of follow-up colonoscopy was contained in the AEMR, regardless of whether the procedure was performed in or out of network.

\section{Data Elements}

The medical records of all patients with a positive FIT or mt-sDNA test during the specified time period were reviewed by a single abstractor. We collected data on demographic characteristics, date of 
the positive stool test, whether a colonoscopy was ordered, and whether a colonoscopy was performed within 6 months of the positive test result. If a follow-up colonoscopy was not completed, using the ambulatory progress notes, we determined whether a colonoscopy was ordered by the provider and any stated reasons why the colonoscopy was not ordered or performed using nonmutually exclusive categories. The reasons for a colonoscopy not ordered were categorized as the positive test being attributed to other reasons, the provider failing to inform the patient of his or her results, the patient having had a colonoscopy within the past year, the patient being referred to a gastroenterology clinic instead of directly ordered a colonoscopy, and the patient refusing the colonoscopy. The reasons a colonoscopy was not performed included the patient refusing or failing to schedule their colonoscopy, the patient cancelling or not completing their appointment, the patient failing to properly complete their preoperative tasks, and the patient having other health issues to prioritize. We also determined whether a colonoscopy was performed but not resulted in the AEMR because it was done out of network. For both positive FIT and positive mt-sDNA, some providers opted to refer the patient for an appointment with a gastroenterology clinic instead of directly ordering a colonoscopy for the patient. Patients for whom the gastroenterologist ordered a colonoscopy that was not completed were included in both the "no-show/cancellation" as well as the "colonoscopy-not-ordered" groups. Finally, those who did not attend their appointment were also included in both the "no-show/cancellation" and "colonoscopy-not-ordered" groups.

\section{Analysis}

Patients for whom the date of stool test (FIT or mt-sDNA) or colonoscopy was missing were excluded. The difference of continuous patient characteristics variables between 2 cohorts of patients with FIT or mt-sDNA screening was made using $t$-test and the association between 2 categorical factors was examined using $\mathrm{c}^{2}$ tests. Time-to-colonoscopy was measured from the date of screening test (FIT or mt-sDNA) to the date of colonoscopy and was censored to the date of last follow-up (December 31, 2019) for those without having colonoscopy. Time to colonoscopy was analyzed using univariate and multivariate Cox proportional hazards models with effect size of predictors esti-mated using hazard ratio (HR) and corresponding $95 \%$ CI. The cumulative rate of having colonoscopy between 2 cohorts of patients was estimated using the Kaplan-Meier method and its difference was examined using log-rank tests. All tests were 2 -sided and $P<.05$ was considered statistically significant.

\section{Results}

\section{Characteristics of Positive FIT and mt-sDNA Patients}

During the study period, we identified a total of 310 patients who received a positive result on their FIT. Two patients were excluded because of missing date of colonoscopy, thus leaving 308 patients eligible for analysis. The mean age was 68.8 years, $47 \%$ of patients were female, and $53 \%$ were male (Table 2). The cohort was majority white $(88 \%)$, with 10\% African American and 1\% or less, Asian, Hispanic, and Native American. Almost 50\% of the cohort had commercial insurance and $43 \%$ had Medicare, while the remainder were either Medicaid (1.9\%), or self pay (1.9\%). Insurance type was unknown in $4.6 \%$. FIT tests were ordered by family medicine specialists in $73 \%$ and by internal medicine providers in 26\%. Approximately $54 \%$ of providers were male and fewer than $5 \%$ were midlevel providers. Of the 308 patients with positive results, $144(46.7 \%)$ patients underwent a colonoscopy within 6 months.

We identified a total of 337 patients who had a positive mt-sDNA test, of whom 14 were excluded from analysis because of missing date of mt-sDNA or colonoscopy. The mean age of the 323 patients was 69.6 years, $63 \%$ were female, and $37 \%$ were male (Table 2). The cohort was majority white (94\%) with 5\% African American and less than 1\% Hispanic. Forty-nine percent of the cohort had Medicare, $46.7 \%$ had commercial insurance, and the remainder were insured by Medicaid (1.6\%) or were self pay $(0.9 \%)$. Insurance type was unknown in $1.9 \%$. More than half of the mt-sDNA tests were ordered by internal medicine providers, $44.3 \%$ by family medicine, and $4.9 \%$ by gastroenterology. Fifty-four percent of tests were ordered by female providers and $6.8 \%$ by midlevel providers. When comparing the 2 groups, the mt-sDNA-positive patients were more likely to be female and have tests ordered by internal medicine and female providers (Table 2). Among the patients with positive testing, 231 (71.5\%) underwent a colonoscopy within 6 months. 
Table 2. Patient Characteristics of Cohort of 631 Patients

\begin{tabular}{|c|c|c|c|c|}
\hline Variables & $\begin{array}{l}\text { All Patients }(\mathrm{n}=631), \text { Mean (STD)/ } \\
\text { Frequency }(\%)\end{array}$ & $\begin{array}{l}\text { Mt-sDNA } \\
(\mathrm{n}=323)\end{array}$ & FIT (n = 308) & $P$ Value* \\
\hline Age (years) & $69.19(10.57)$ & $69.55(7.95)$ & $68.82(12.76)$ & .39 \\
\hline \multicolumn{5}{|l|}{ Sex } \\
\hline Female & $349(55.3)$ & $205(63.47)$ & $144(46.75)$ & \multirow[t]{2}{*}{$<.0001$} \\
\hline Male & $282(44.7)$ & $118(41.84)$ & $164(53.25)$ & \\
\hline \multicolumn{5}{|l|}{ Hispanic } \\
\hline No & $573(99.13))$ & $285(99.30)$ & $288(98.97)$ & \multirow[t]{2}{*}{.66} \\
\hline Yes & $5(0.87)$ & $2(0.70)$ & $3(1.03)$ & \\
\hline \multicolumn{5}{|l|}{ Race } \\
\hline Asian & $3(0.49)$ & $0(0.00)$ & $3(0.99)$ & \multirow{6}{*}{.064} \\
\hline Black & $48(7.77)$ & $17(5.41)$ & $31(10.20)$ & \\
\hline Other & $1(0.16)$ & $0(0.00)$ & $1(0.33)$ & \\
\hline White & $563(91.10)$ & $296(94.27)$ & $267(87.83)$ & \\
\hline Hispanic & $2(0.32)$ & $1(0.32)$ & $1(0.33)$ & \\
\hline Native & $1(0.16)$ & $0(0.00)$ & $1(0.33)$ & \\
\hline \multicolumn{5}{|l|}{ Insurance } \\
\hline Commercial & $301(47.7)$ & $151(46.7)$ & $150(48.7)$ & \multirow{5}{*}{.18} \\
\hline Medicaid & $11(1.7)$ & $5(1.6)$ & $6(1.9)$ & \\
\hline Medicare & $290(46)$ & $158(48.9)$ & $132(42.9)$ & \\
\hline Self-pay & $9(1.4)$ & $3(0.9)$ & $6(1.9)$ & \\
\hline Unknown & $20(3.2)$ & $6(1.9)$ & $14(4.6)$ & \\
\hline \multicolumn{5}{|l|}{ Specialty } \\
\hline Family Medicine & $368(58.3)$ & $143(44.3)$ & $225(73)$ & \multirow{4}{*}{$<.0001$} \\
\hline Internal Medicine & $242(38.3)$ & $163(50.5)$ & $79(25.7)$ & \\
\hline GI & $20(3.2)$ & $16(4.9)$ & $4(1.3)$ & \\
\hline Gynecology & $1(0.2)$ & $1(0.3)$ & $0(0)$ & \\
\hline \multicolumn{5}{|l|}{ Provider Gender } \\
\hline Female & $317(50.2)$ & $175(54.2)$ & $142(26.1)$ & \multirow[t]{2}{*}{.43} \\
\hline Male & $314(49.8)$ & $148(45.8)$ & $166(53.9)$ & \\
\hline \multicolumn{5}{|l|}{ Mid-Level Provider } \\
\hline Yes & $37(5.9)$ & $22(6.8)$ & $15(4.9)$ & \multirow[t]{2}{*}{.3} \\
\hline No (physician) & $594(94.1)$ & $301(93.2)$ & $293(95.1)$ & \\
\hline
\end{tabular}

${ }^{*} P$ value was based on the comparison between two cohorts (FIT, mt-sDNA) of patients.

GI, gastroenterology; FIT, fecal immunochemical testin; STD, standard deviation.

\section{Barriers to Follow-up in Positive FIT and mt-sDNA Patients}

The electronic medical records of the 164 patients without colonoscopy after a positive FIT were reviewed to determine reasons for lack of followup. In 135 patients, the provider did not order a colonoscopy. For many (23.8\%), the positive FIT was attributed to other causes than possible colorectal pathology. For $29.3 \%$ of the patients, instead of directly ordering the colonoscopy, the provider referred the patients to a gastroenterology (GI) clinic appointment which they did not keep. Another $14.6 \%$ of the patients had a colonoscopy (within the past year) and were determined to not need another. Other reasons included a presumed false-positive result (1.8\%), and the patient refusing the colonoscopy $(7.4 \%)$. There were also several instances $(16 \%)$ where the reasoning behind the provider failing to order a colonoscopy after a positive FIT was not indicated in the patients' medical record. In these instances, the provider did not acknowledge the patient's positive result in subsequent visits, or the patient did not return for any future visits to the provider (Table 3). For the 30 patients for whom a colonoscopy was ordered but not performed, most did not show or cancelled their appointment. Other reasons included patients refusing or not scheduling the procedure and the patients having other health issues to prioritize (Table 3). Overall, system level factors were iden- 
Table 3. Reasons for Lack of Follow-up after Positive mt-sDNA or FIT*

\begin{tabular}{lcc}
\hline & $\begin{array}{c}\text { FIT } \\
(\mathrm{n}=164) \\
\text { Frequency } \\
(\%)\end{array}$ & $\begin{array}{c}\text { Mt-sDNA } \\
(\mathrm{n}=92) \\
\text { Frequency }(\%)\end{array}$ \\
\hline System & & \\
GI clinic referral & $48(29.3)$ & $11(12.0)$ \\
Colonoscopy not scheduled & $5(3.0)$ & $17(18.5)$ \\
Total & $\mathbf{5 3 ( 3 2 . 3 )}$ & $\mathbf{2 8 ( 3 0 . 4 )}$ \\
Provider level & & \\
Attributed to false positive & $3(1.8)$ & $0(0.0)$ \\
Failure to inform patient & $0(0.0)$ & $7(7.6)$ \\
Attributed to other reasons & $39(23.8)$ & $1(1.1)$ \\
Recent colonoscopy done & $24(14.6)$ & $1(1.1)$ \\
Other health issue to prioritize & $8(4.9)$ & $11(12.0)$ \\
Unknown & $21(12.8)$ & $20(21.7)$ \\
Total & $\mathbf{9 5 ( 5 7 . 9 )}$ & $\mathbf{4 0}(\mathbf{4 3 . 5})$ \\
Patient level & & \\
Patient refused (before & $8(4.9)$ & $8(8.7)$ \\
ordered) & & \\
Patient refused (after ordered) & $4(2.5)$ & $15(16.3)$ \\
No show/cancellation & $42(25.6)$ & $26(28.3)$ \\
Did not complete bowel prep & $0(0)$ & $1(1.1)$ \\
Other reason & $6(3.7)$ & $3(3.3)$ \\
Total & $\mathbf{6 0 ( 3 6 . 6 )}$ & $\mathbf{5 3 ( 5 7 . 6 )}$ \\
\hline
\end{tabular}

*Determined by medical record review.

FIT, fecal immunochemical testing; GI, gastroenterology.

tified in $22.3 \%$, provider-level factors in $57.9 \%$, and patient-related factors in $36.6 \%$.

Review of the electronic health records of the 92 patients $(28.5 \%)$ who did not receive a colonoscopy after a positive mt-sDNA indicated several reasons for this lack of completion. For 47 patients $(51 \%)$, the provider did not order a colonoscopy. In $12 \%$ of cases, the patient was referred for a GI clinic appointment that they did not keep. In addition, $25 \%$ of patients expressed unwillingness to undergo colonoscopy and in $7.6 \%$ the provider failed to inform the patient of their mt-sDNA results. Other reasons included the provider attributing the positive mt-sDNA test to other reasons $(1.1 \%)$, and the patient having a colonoscopy within the past year $(1.1 \%)$. As in the case with positive FIT tests, there were instances where there was no apparent reason for the provider not ordering a colonoscopy after a positive mt-sDNA test (Table 3). A colonoscopy was ordered and not completed in 45 patients (49\%). The most common reasons for the lack of colonoscopy completion were the patient refusing or not scheduling the colonoscopy and the patient cancelling or not showing up to their colonoscopy appointment. Other reasons included the patient having other health issues to prioritize and the patient not completing the bowel preparation (Table 3). Overall system-level factors were implicated in $30.4 \%$, provider related factors in $43.5 \%$, and patient related factors in $57.6 \%$.

\section{Follow-up in FIT Versus mt-sDNA-Positive Patients}

We compared time to follow-up colonoscopy among patients with positive FIT versus mt-sDNA (Table 4). In this analysis, compared with FIT, the hazard ratio of having colonoscopy after mt-sDNA was 1.83 (95\% CI, 1.48-2.25). No other sociodemographic characteristics were associated with time to colonoscopy, though we did find a positive association with female providers (HR, 1.30; 95\% CI, 1.06-1.60). These differences are also shown graphically in an unadjusted Kaplan Meier analysis with a median time to colonoscopy of 2.2 months for mt-sDNA and $>6$ months for FIT (Figure 1). In a multivariable Cox proportional hazards model adjusting for age, gender, race, type of insurance and provider, gender, similar differences were observed, with a significant difference in time to colonoscopy observed in positive mt-sDNA patients compared with positive FIT patients (HR, 1.75 ; 95\% CI, 1.40-2.19) (Table 4). However, there was no longer an association with provider gender. After excluding the 25 patients (24 FIT, $1 \mathrm{mt}$ sDNA) who were not referred for colonoscopy because it had already been recently performed, follow-up was still higher in mt-sDNA than FIT (71.7\% vs $50.7 \%$, respectively; $P \leq .001$ ).

\section{Yield of Colonoscopy with Positive FIT or mt-sDNA}

Among the 144 patients with a positive FIT who underwent colonoscopy, precancerous or malignant lesions were found in $70(48.6 \%)$. The most advanced finding included adenocarcinoma $(n=5)$, adenomas with high-grade dysplasia $(\mathrm{n}=5)$, villous or tubulovillous adenomas $(\mathrm{n}=11)$, sessile serrated adenomas $(n=9)$ and tubular adenomas $(n=40)$. For the 231 patients with positive mt-sDNA testing, the yield was even higher $(77.1 \%)$. The most advanced finding included adenocarcinoma $(n=8)$, adenomas with high-grade dysplasia $(n=9)$, villous or tubulovillous adenomas $(\mathrm{n}=19)$, sessile serrated adenomas $(n=33)$, and tubular adenomas $(n=109)$. 
Table 4. Cox Regression Analysis on Time-to-Colonoscopy

\begin{tabular}{|c|c|c|c|c|c|c|}
\hline \multirow[b]{2}{*}{ Variables } & \multicolumn{3}{|c|}{ Univariate Cox Regression } & \multicolumn{3}{|c|}{ Multivariable Cox Regression } \\
\hline & HR & $95 \% \mathrm{CI}$ of $\mathrm{HR}$ & $P$ value & HR & $95 \% \mathrm{CI}$ of $\mathrm{HR}$ & $P$ Value \\
\hline Age (per year increase) & 0.99 & $0.98,1.00$ & .13 & 0.99 & $0.98,1.00$ & .181 \\
\hline Sex (female vs male) & 1.17 & $0.95,1.43$ & .14 & 1.09 & $0.83,1.32$ & .717 \\
\hline Race (Black vs White) & 1.30 & $0.91,1.85$ & .16 & 1.38 & $0.95,2.00$ & .089 \\
\hline Insurance (commercial vs Medicare) & 1.12 & $0.91,1.38$ & .29 & 1.10 & $0.87,1.39$ & .411 \\
\hline Screening test method (mt-sDNA vs FIT) & 1.83 & $1.48,2.25$ & $<.0001$ & 1.75 & $1.40,2.19$ & $<.0001$ \\
\hline Specialty (FM vs IM) & 0.87 & $0.71,1.08$ & .202 & & & \\
\hline Provider gender (female vs male) & 1.30 & $1.06,1.60$ & .01 & 1.19 & $0.95,1.50$ & .125 \\
\hline Mid-level provider (yes vs no) & 1.16 & $0.77,1.75$ & .483 & & & \\
\hline
\end{tabular}

FIT, fecal immunochemical testing; FM, family medicine; HR, hazard ratio; IM, internal medicine; CI, confidence interval.

\section{Discussion}

CRC screening is recommended for all adults and has been shown to decrease the incidence and mortality from CRC. Current guidelines offer patients and providers a choice between different options. $^{2-4}$ The stool-based tests, including FIT

Figure 1. Kaplan-Meier estimation of time-to-colonoscopy of patients who had FIT test and who had mt-sDNA. The median time-to-colonoscopy for patients FIT was N/A (50\% rate was not reached for this group). The median (95\% CI) time-to-colonoscopy for patients who had mt-sDNA was 2.16 (95\% CI, 1.80-2.52) months. Abbreviation: FIT, fecal immunochemical testing.

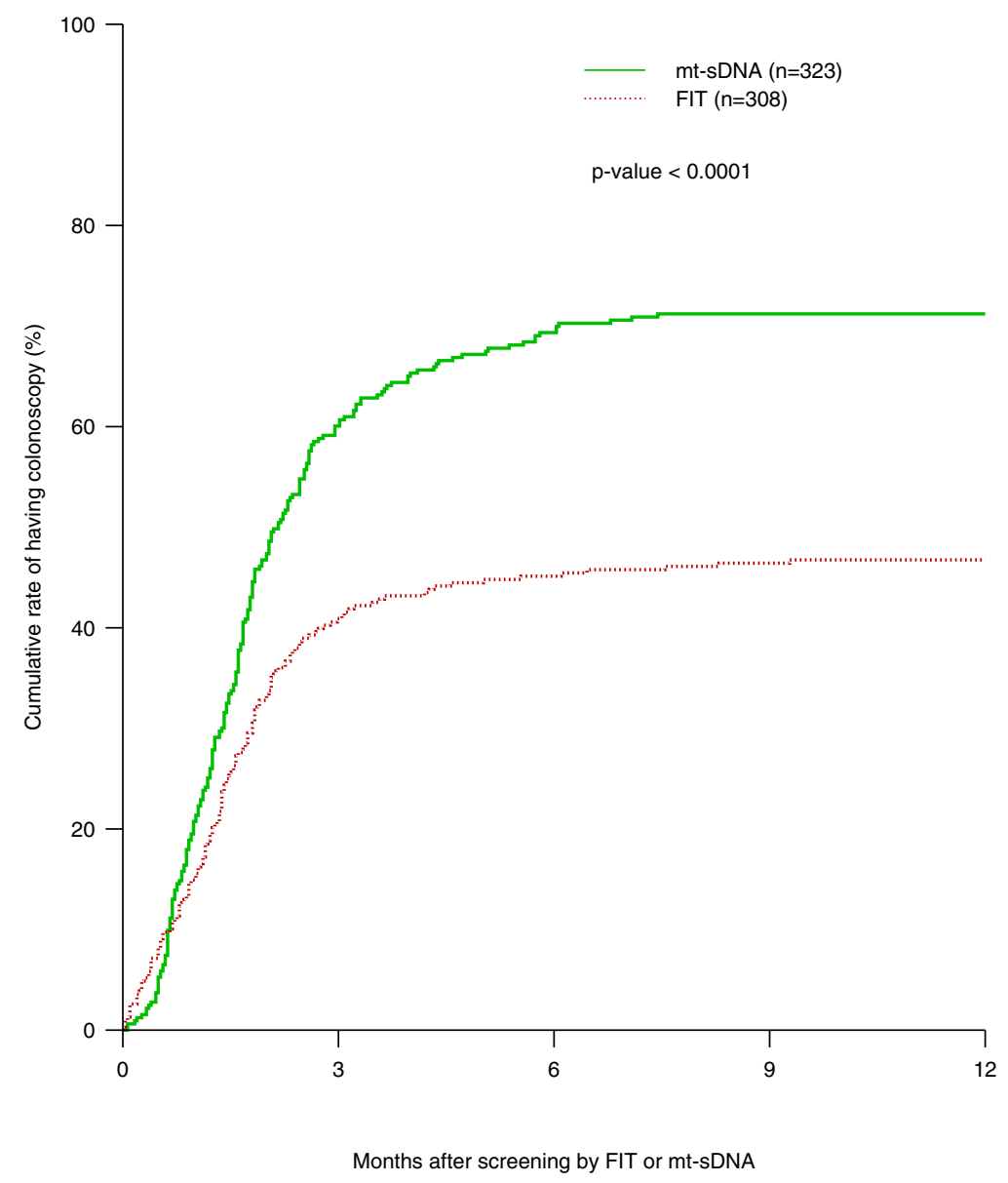


and mt-sDNA are specifically targeted for patients who prefer an alternative to colonoscopy or in whom colonoscopy would be difficult. The positive predictive value (PPV) of FIT testing for cancer is $2 \%$ to $11 \%$ and in a retrospective analysis, the PPV for detecting advanced neoplasia (advanced adenomas or cancer) was $24 \% .{ }^{19}$ In the largest clinical trial of mt-sDNA testing, the PPV for advanced neoplasia was $24 \% .{ }^{20}$ Thus, it is important that patients with positive testing undergo appropriate evaluation with colonoscopy to detect and remove underlying neoplasia. The US Multisociety Task Force on Colorectal Cancer has recommended a minimum of $80 \%$ of patients have a follow-up colonoscopy after a positive FIT $^{6}$ though similar guidelines for mt-sDNA are not in place.

In our study, which included patients from a vertically integrated health system, we found that compliance with follow-up colonoscopy after a positive FIT was only $46.7 \%$ and although compliance was higher after a positive mt-sDNA test, it was $71.5 \%$, not nearly the preferred $100 \%$. The colonoscopy frequency after positive FIT was in the range of previous reports. ${ }^{7-11}$ One small study in a multispecialty group practice found close to $100 \%$ followup after positive mt-sDNA, ${ }^{12}$ but the findings may not be generalizable to other settings. Moreover, we found that a combination of provider and health system issues were more frequent barriers to colonoscopy completion than patient-related factors, especially after positive FIT. Although this finding was in contrast with a recent VA-based study that found patient cancellation of colonoscopy was the most frequent reason for lack of colonoscopy after positive stool testing, ${ }^{9}$ they also reported system and provider factors as common deterrents. In a qualitative study from Ontario, Canada, attribution of a positive FIT to other causes and patient fear of colonoscopy were the 2 most common identifiable reasons for lack of follow-up. ${ }^{18} \mathrm{~A}$ recently published qualitative that included patients from federally qualified health centers also identified fear as a barrier but also determined that communication about the need for colonoscopy, transportation and cost of the procedure were additional obstacles. ${ }^{21}$

Whereas patients who are inherently reluctant to have a colonoscopy may have self-selected themselves for noninvasive screening options, we found several additional barriers to completion. A common reason for lack of compliance with colonoscopy in our study was that despite having a direct endoscopy referral system, providers ordered a gastroenterology clinic referral before colonoscopy, which the patient often did not keep. Although for certain patients (ie, those on anticoagulants or with a history of anesthetic reactions or inflammatory bowel disease) the gastroenterologist may have required visits before undertaking colonoscopy, in many cases the visit may not have been necessary and created another level of complexity. In addition, the extra appointment may not have been feasible for certain patients who could not miss work or other responsibilities or had transportation difficulties. In contrast, a VA based study found that referral to gastroenterology actually facilitated colonoscopy completion, ${ }^{10}$ which suggests that barriers to colonoscopy in an individual health care setting should be assessed before proposing solutions. We also noted that although some providers did not refer patients because of concerns of competing comorbidities or evidence of recent colonoscopy, these issues may not have been addressed before stool testing. Another potential barrier is the out-of-pocket cost associated with colonoscopy, which is considered diagnostic if performed as a result of a positive stool test. Our database did not include the out-of-pocket costs that patients faced.

Although we found a higher rate of follow-up after positive mt-sDNA testing than with positive FIT testing, reasons for lack of follow-up were generally similar between the 2 modalities. Whereas the reasons for this difference in follow-up frequency could not be determined from this study, the differences persisted even after adjustment for demographic characteristics such as insurance coverage. However, there may also be unmeasured physician and/or patient biases as to which test was ordered. If mt-sDNA was more likely to be ordered than FIT for patients who were more motivated at baseline to complete the follow-up that could account for some of the observed differences. In addition, for both FIT and mt-sDNA, colonoscopy performed as a follow-up to a positive stool test is generally considered to be diagnostic as opposed to screening and potentially subject to coinsurance; this could create an additional barrier for lowincome patients. Physicians may have also ordered FIT testing as a confirmatory test to assess gastrointestinal blood loss as opposed to a screening test. In addition, the manufacturer of mt-sDNA employs a patient navigator program that encourages patients to return the kits. Although the navigators do not facilitate follow-up with colonoscopy after 
positive tests, they may have previously emphasized the importance of appropriate follow-up.

Multiple clinical trials have also evaluated interventions to improve follow-up of positive FIT tests $^{22}$ though similar studies have not been performed for mt-sDNA. Based on a systematic review, it seems that the use of patient navigators and providing reminders or performance data to providers have the greatest efficacy, though studies were often prone to bias and the effect size was only moderate. In Kaiser Northern California, a multistep system-level strategy that included standardized tracking, reminders and navigator use improved colonoscopy completion from $73 \%$ to $85 \% .^{23}$ A consensus conference also supported patient navigation as an effective strategy to improve follow-up with colonoscopy. ${ }^{24}$ Recent work in an indigent patient population in New Hampshire has found to use of navigators to promote colonoscopy screening was effective in more than $97 \%$ of patients ${ }^{25}$ and cost effective in both public health programs and endoscopy center outreach. $^{26}$

We recognize several strengths and limitations of our study. The sample includes patients from a large, diverse patient population from a Midwestern academic health care system with an electronic medical record system that encompasses office notes, test results, and procedures. Limitations include ambiguity about reasons for lack of follow-up if not documented in the clinical notes, as well as potentially missing procedure notes if a colonoscopy was performed at an outside facility, not scanned into the patient's chart and not documented in an office note. This was a particular concern for mt-sDNA. In addition, in certain scenarios the patient may not have known about the referral to gastroenterology or colonoscopy and could not be contacted. We also could not measure certain patient characteristics that may have been associated with follow-up, including educational level, income, and language barriers.

In summary, follow-up colonoscopy after positive stool testing was higher for mt-sDNA than FIT, which should be considered in the programmatic evaluation of noninvasive screening options. However, follow-up after both tests was less than ideal. In our cohort, factors attributable to the provider and system were as frequently encountered as reasons for lack of follow-up colonoscopy as patient factors. These findings reinforce the need for multi-level interventions to improve follow-up. ${ }^{27}$
To see this article online, please go to: http://jabfm.org/content/ 34/1/61.full.

\section{References}

1. Siegel RL, Miller KD, Jemal A. Cancer statistics, 2020. CA Cancer J Clin 2020;70:7-30.

2. Rex DK, Boland CR, Dominitz JA. Colorectal cancer screening: recommendations for physicians and patients from the U.S. Multisociety Task Force on Colorectal Cancer. Am J Gastroenterol 2017; 112:1016-30.

3. Bibbins-Domingo K, Grossman DC, Curry SJ, et al. Screening for colorectal cancer: US Preventive Services Task Force recommendation. JAMA 2016;315: 2564-75.

4. Smith RA, Andrews KS, Brooks D, et al. Cancer screening in the United States, 2019: a review of current American Cancer Society guidelines and current issues in cancer screening. CA Cancer J Clin 2019;69:184-210.

5. Robertson DJ, Imperiale TF. Stool testing for colorectal cancer screening. Gastroenterology 2015;149: 1286-93.

6. Robertson DJ, Lee JK, Boland CR, et al. Recommendations on fecal immunochemical testing to screen for colorectal neoplasia: a consensus statement by the US Multi-Society Task Force on Colorectal Cancer. Am J Gastroenterol 2017;112:37-53.

7. Tosteson ANA, Beaber EF, Tiro J, et al. Variation in screening abnormality rates and follow-up of breast, cervical and colorectal screening within the PROSPR Consortium. J Gen Intern Med 2016;31:372-9.

8. Etzioni DA, Yano EM, Rubinstein LV, et al. Measuring the quality of colorectal cancer screening: the importance of follow-up. Dis Col Rect 2006;49:1002-10.

9. May FP, Yano EM, Provenzale D, et al. Barriers to colonoscopies for patients with positive results from fecal immunochemical tests during colorectal cancer screening. Clin Gastroenterol Hepatol 2019;17: 469-76.

10. Fisher DA, Jeffreys A, Coffman CJ, Fasanella K. Barriers to full colon evaluation for a positive fecal occult blood test. Cancer Epidemiol Biomarkers Prev 2006;15:1232-5.

11. Turner B, Myers RE, Hyslop T, et al. Physician and patient factors associated with ordering a colon evaluation after a positive fecal occult blood test. J Gen Intern Med 2003;18:357-63.

12. Prince $M$, Lester L, Chiniwala R, Berger B. Multitarget stool DNA tests increases colorectal cancer screening among previously noncompliant Medicare patients. World J Gastroenterol 2017; 23:464-71.

13. Corley DA, Jensen CD, Quinn VP, et al. Association between time to colonoscopy after a positive fecal test result and risk of colorectal 
cancer and cancer stage at diagnosis. JAMA 2017;317:1631-41.

14. Doubeni CA, Gabler NB, Wheeler CM, et al. Timely follow-up of positive cancer screening results: a systematic review and recommendations from the PROSPR consortium. CA Cancer J Clin 2018;68:199-216.

15. Meester RGS, Zauber AG, Doubeni CA, et al. Consequences of increasing time to colonoscopy examination after positive result from fecal colorectal cancer screening test. Clin Gastroenterol Hepatol 2016;14:1445-51.

16. Issaka RB, Singh $\mathrm{MH}$, Oshima SM, et al. Inadequate utilization of diagnostic colonoscopy following abnormal FIT results in an integrated safety-net system. Am J Gastroenterol 2017;112:375-82.

17. Martin J, Halm EA, Tiro JA, et al. Reasons for lack of diagnostic colonoscopy after positive result on fecal immunochemical test in a safety-net health system. Am J Med 2017;130:93.e193-e197.

18. Llovet D, Serenity M, Conn LG, et al. Reasons for lack of follow-up colonoscopy among persons with a positive fecal occult blood test result: a qualitative study. Am J Gastroenterol 2018;113:1872-80.

19. Alsayid M, Singh MH, Issaka R, et al. Yield of colonoscopy after a positive result from a fecal immunochemical test OC-light. Clin Gastroenterol Hepatol 2018;16:1593-7.

20. Imperiale TF, Ransohoff DF, Itzkowitz SH, et al. Multitarget stool DNA testing for colorectal cancer screening. N Engl J Med 2014;370:1287-97.
21. Schneider JL, Rivelli JS, Gruss I, et al. Barriers and facilitators to timely colonoscopy completion for safety net clinic patients. Am J Health Behav 2020;44:460-72.

22. Selby K, Baumgartner C, Levin TR, et al. Interventions to improve follow-up on positive results on fecal blood tests: a systematic review. Ann Intern Med 2017;167:565-75.

23. Selby K, Jensen CD, Zhao WK, et al. Strategies to improve follow-up after positive fecal immunochemical tests in a community-based setting: a mixed-methods study. Clin Transl Gastroenterol 2019;10:e00010.

24. Gupta S, Coronado GD, Argenbright K, et al. Mailed fecal immunochemical test outreach for colorectal cancer screening: summary of a Centers for Disease Control and Prevention-sponsored summit. CA Cancer J Clin 2020;70:283-98.

25. DeGroff A, Gressard L, Glover-Kudon R, et al. Assessing the implementation of a patient navigation intervention for colonoscopy screening. BMC Health Serv Res 2019;19:803.

26. Rice K, Sharma K, Li C, et al. Cost-effectiveness of a patient navigation intervention to increase colonoscopy screening among low-income adults in New Hampshire. Cancer 2019;125:601-9.

27. Zapka J, Taplin SH, Price RA, et al. Factors in quality care-The case of follow-up to abnormal cancer screening tests-Problems in the steps and interfaces of care. J Natl Cancer Inst Monogr 2010;2010:58-71. 